\title{
Connectivity of diagonal products of Baire one functions
}

by

\author{
A. Maliszewski (Bydgoszcz)
}

\begin{abstract}
We characterize those Baire one functions $f$ for which the diagonal product $x \mapsto(f(x), g(x))$ has a connected graph whenever $g$ is approximately continuous or is a derivative.
\end{abstract}

I. Introduction. It is well known for a long time that the product of two derivatives need not be a derivative. Moreover, the characteristic function of every closed set can be written as the product of two bounded derivatives [2]. So the graph of the product of two derivatives need not be connected, whence the graph of the diagonal product of two derivatives need not be connected. On the other hand, it is well known (and easy to prove) that the product of a bounded approximately continuous function with a bounded derivative is a derivative again, so its graph is connected. However, the graph of the diagonal product of a bounded approximately continuous function with a bounded derivative is not necessarily connected. In 1963 Neugebauer [7] constructed a bounded approximately continuous function $f$ and a bounded derivative $g$ such that $f(0)=g(0)=0$ and $[f(x)]^{2}+[g(x)]^{2} \geq 1 / 4$ for $x \neq 0$, so the graph of $f \triangle g$ is not connected. In this paper our aim is to characterize those Baire one functions $f$ for which the diagonal product $f \triangle g$ has a connected graph whenever $g$ is approximately continuous or is a derivative.

II. Preliminaries. The real line $(-\infty, \infty)$ is denoted by $\mathbb{R}$ and the set of positive integers by $\mathbb{N}$. The only measure used is Lebesgue measure in $\mathbb{R}$ and all integrals are Lebesgue integrals. For each set $A \subset \mathbb{R}$, int $A$ denotes its (Euclidean) interior, $\operatorname{cl} A$ its closure, fr $A$ its boundary and $|A|$ its outer measure.

1991 Mathematics Subject Classification: Primary 26A15; Secondary 54C08.

Key words and phrases: Darboux function, peripheral continuity, approximate continuity, derivative, Baire one function, diagonal product.

Supported by a KBN Research Grant 2114491 01, 1992-94. 
The word function means a mapping from $\mathbb{R}$ into $\mathbb{R}$ unless otherwise explicitly stated. The graph of a function $f: X \rightarrow Y$ will be denoted by $\Gamma(f)$. We denote by $\mathcal{D}$ the family of all derivatives. If $X$ and $Y$ are metric spaces, then the family of all Baire one functions from $X$ into $Y$ (i.e., pointwise limits of sequences of continuous functions) will be denoted by $\mathcal{B}_{1}(X, Y)$. We write $\mathcal{B}_{1}$ for $\mathcal{B}_{1}(\mathbb{R}, \mathbb{R})$.

The terms $d$-closed, $d$-interior ( $d$-int) etc. will refer to the Denjoy topology (density topology) on $\mathbb{R}$. (See, e.g., [3], [9], [5].) We say that a function $f$ is approximately continuous if it is continuous relative to the Denjoy topology. The family of all approximately continuous functions will be denoted by $\mathcal{C}_{\text {ap }}$. Recall that each element of $\mathcal{C}_{\text {ap }}$ is a Baire one function and each bounded element of $\mathcal{C}_{\text {ap }}$ is a derivative (see, e.g., [1]).

We denote by $b$ the family of all bounded functions. We drop the " $\cap$ " sign between classes of functions, e.g., $b \mathcal{C}_{\text {ap }}$ denotes the family of all bounded approximately continuous functions.

We denote by $\mathcal{C}$ the family of all continuous functions, by $\mathcal{C}_{\text {a.e. }}$ the family of all functions which are continuous almost everywhere and by $\mathcal{C}_{0}$ the family of all functions which are continuous except possibly at one point. It is well known that elements of $\mathcal{C}_{\text {ap }} \mathcal{C}_{\text {a.e. }}$ are exactly those functions which are continuous with respect to the so-called a.e.-topology [8]. Recall that a set $A \subset \mathbb{R}$ is a.e.-open if and only if it is $d$-open and $|A|=|\operatorname{int} A|$.

For each set $T \subset \mathbb{R}$ and $a, b \in \mathbb{R}, a<b$, we denote by $\varphi_{T}(a, b)$ the measure of the greatest interval $J$ contained in $(a, b) \backslash T$ if any such interval exists, and 0 otherwise. We say that a set $T \subset \mathbb{R}$ is porous at $x \in \mathbb{R}$ from the left if

$$
\limsup _{\eta \rightarrow 0^{+}} \frac{\varphi_{T}(x-\eta, x)}{\eta}>0 .
$$

Being porous from the right is defined similarly.

We say that a set $T \subset \mathbb{R}$ is non-degenerate at $x \in \mathbb{R}$ from the left if

$$
\limsup _{\eta \rightarrow 0^{+}} \frac{|T \cap(x-\eta, x)|}{\eta}>0 .
$$

Non-degeneracy from the right is defined similarly.

Let $Y$ be a topological space and let $f: \mathbb{R} \rightarrow Y$. We say that

- $f$ is a Darboux function if the $f$-image of each interval is connected,

- $f$ is peripherally continuous if

$$
f^{-1}(U) \cap(x-\eta, x) \neq \emptyset \neq f^{-1}(U) \cap(x, x+\eta)
$$

for each $x \in \mathbb{R}$, each neighborhood $U \subset Y$ of $f(x)$ and each $\eta>0$.

Given two functions $f, g: \mathbb{R} \rightarrow \mathbb{R}$, we define their diagonal product $f \triangle g: \mathbb{R} \rightarrow \mathbb{R}^{2}$ by $(f \triangle g)(x)=(f(x), g(x))$. 
Let $\mathcal{A} \subset \mathcal{B}_{1}$. The maximal diagonal class of $\mathcal{A}$ with respect to connectivity is the family $\mathcal{M}_{\triangle}(\mathcal{A})$ of those Baire one functions whose diagonal product with each function from $\mathcal{A}$ has a connected graph.

III. Auxiliary lemmas. There are many conditions which are equivalent to the connectivity of the graph of a function $f \in \mathcal{B}_{1}$ (cf. [1, Theorem 1.1, p. 9]). For our purpose we will generalize two of them.

Lemma 1. Assume that $Y$ is a $T_{1}$-space and $f: \mathbb{R} \rightarrow Y$ is a Darboux function. Then $f$ is peripherally continuous.

Proof. Suppose that $f$ is not peripherally continuous from the left at some $x \in \mathbb{R}$. Let a neighborhood $U \subset Y$ of $f(x)$ and $\eta>0$ be such that $f^{-1}(U) \cap(x-\eta, x)=\emptyset$. Set $A=f((x-\eta, x])$ and $V=Y \backslash\{f(x)\}$. Since $Y$ is a $T_{1}$-space, $V$ is open. Hence $A_{1}=A \cap U=\{f(x)\}$ and $A_{2}=A \cap V$ are open in $A$, disjoint and non-empty, and $A_{1} \cup A_{2}=A$. So $A$ is not connected and $f$ is not a Darboux function, which completes the proof.

Lemma 2. Assume that $Y$ is a metric space and $f \in \mathcal{B}_{1}(\mathbb{R}, Y)$ is peripherally continuous. Then the graph of $f$ is connected.

Proof. Suppose that $\Gamma(f)$ is not connected. Then there exist disjoint non-empty sets $E_{1}, E_{2} \subset \Gamma(f)$, open in $\Gamma(f)$, such that $E_{1} \cup E_{2}=\Gamma(f)$. For $j \in\{1,2\}$ let $D_{j}$ be the preimage of $E_{j}$ under the map $x \mapsto(x, f(x))$. Then $D_{1}, D_{2}$ are disjoint, non-empty and $D_{1} \cup D_{2}=\mathbb{R}$, so fr $D_{1} \neq \emptyset$. By [4] (§31.X.5, p. 397), the set of points of continuity of $f \mid$ fr $D_{1}$ is nonempty. Let $x$ be one. By symmetry, we may assume that $x \in D_{1}$ and that $(x-\tau, x) \cap D_{2} \neq \emptyset$ for each $\tau>0$. Since $E_{1}$ and $E_{2}$ are disjoint and open in $\Gamma(f)$, there exists a neighborhood $U \subset Y$ of $f(x)$ and a $\tau_{1}>0$ such that $f(t) \notin U$ for $t \in\left(x-\tau_{1}, x+\tau_{1}\right) \cap D_{2}$. Since $f \mid$ fr $D_{1}$ is continuous at $x$, there exists a $\tau_{2} \in\left(0, \tau_{1}\right)$ such that $f(t) \in U$ for each $t \in\left(x-\tau_{2}, x\right] \cap$ fr $D_{1}$. So $D_{2} \cap\left(x-\tau_{2}, x\right) \cap$ fr $D_{1}=\emptyset$, whence $D_{2} \cap\left(x-\tau_{2}, x\right) \neq \emptyset$ is open. Let $(a, b)$ be a component of $D_{2} \cap\left(x-\tau_{2}, x\right)$. Then $b \in\left(x-\tau_{2}, x\right] \cap$ fr $D_{1}$ and $f(b) \in U$. But $f(t) \notin U$ for $t \in(a, b)$. So $f$ is not peripherally continuous at $b$ from the left, contrary to the assumption.

The next lemma follows easily from the definitions.

Lemma 3. Assume that $Y$ is an arbitrary topological space and that the graph of $f: \mathbb{R} \rightarrow Y$ is connected. Then $f$ is a Darboux function.

From the above three lemmas we get the following theorem.

TheOREM 4. Let $Y$ be a metric space and $f \in \mathcal{B}_{1}(\mathbb{R}, Y)$. Then the following conditions are equivalent:

(A) $f$ is a Darboux function, 
(B) $f$ is peripherally continuous,

(C) $f$ has a connected graph.

We will also need the following lemma.

Lemma 5 [6, Lemma 9]. Assume that $\left\{J_{n}: n \in \mathbb{N}\right\}$ is a family of nonoverlapping intervals and $\left(r_{n}\right)$ is a sequence of non-negative numbers with $\sum_{n=1}^{\infty} r_{n}<\infty$. Then there exists a sequence $\left(t_{n}\right)$ such that $\left|t_{n}\right|=1$ for each $n \in \mathbb{N}$ and for every interval $I \subset \mathbb{R}$,

$$
\left|\sum_{J_{n} \subset I} t_{n} r_{n}\right| \leq 2 \sup \left\{r_{n}: J_{n} \subset I\right\} .
$$

IV. Main results. First we will deal with the family $\mathcal{C}_{\text {ap }}$.

Proposition 6. Let $f \in \mathcal{B}_{1}$. Then the following conditions are equivalent:

(i) $f \in \mathcal{M}_{\triangle}\left(\mathcal{C}_{\text {ap }}\right)$,

(ii) $f \in \mathcal{M}_{\triangle}\left(\mathrm{b} \mathcal{C}_{\text {ap }}\right)$,

(iii) for each $x \in \mathbb{R}$ and each $\varepsilon>0$ the set $f^{-1}([f(x)-\varepsilon, f(x)+\varepsilon])$ is bilaterally non-degenerate at $x$.

Proof. The implication (i) $\Rightarrow$ (ii) is obvious.

(ii) $\Rightarrow$ (iii). Suppose that for some $x \in \mathbb{R}$ and some $\varepsilon>0, C$ is not nondegenerate at $x$ from the left, where $C=f^{-1}([f(x)-\varepsilon, f(x)+\varepsilon])$. For each $n \in \mathbb{N}$ set $x_{n}=x-1 / n, I_{n}=\left[x_{n}, x_{n+1}\right]$ and $A_{n}=I_{n} \backslash C$, find a closed set $B_{n} \subset d$-int $A_{n}$ such that $\left|A_{n} \backslash B_{n}\right| \leq\left|A_{n}\right| / n$, and use Lemma 12 of [10] to find an approximately continuous function $g_{n}$ such that $0 \leq g_{n} \leq 1$ on $\mathbb{R}$, $g_{n}=1$ off $A_{n}$ and $g_{n}=0$ on $B_{n}$. Define

$$
g(t)= \begin{cases}0 & \text { if } t \leq x_{1} \text { or } t \geq x \\ \varepsilon g_{n}(t) & \text { if } t \in I_{n}, n \in \mathbb{N}\end{cases}
$$

Then clearly $g$ is bounded and approximately continuous except possibly at $x$, and it is continuous at $x$ from the right. Moreover, for each $t \in\left[x_{n}, x_{n+1}\right]$,

$$
\begin{aligned}
\frac{|\{z \in[t, x]: g(z)-g(x)=0\}|}{x-t} & \geq \frac{1}{x-x_{n}}\left|\bigcup_{k=n+1}^{\infty} B_{k}\right| \\
& \geq \frac{n}{n+1}\left(1-\frac{1}{n+1}\right) \frac{\left|\left[x_{n+1}, x\right] \backslash C\right|}{x-x_{n+1}} .
\end{aligned}
$$

So $g$ is approximately continuous at $x$ from the left. But for each $t \in\left[x_{1}, x\right)$ either $t \notin \bigcup_{n=1}^{\infty} A_{n}$, so that $g(t)=g(t)-g(x)=\varepsilon$, or $t \in \bigcup_{n=1}^{\infty} A_{n}$, and then $t \notin C$ and $|f(t)-f(x)|>\varepsilon$. Hence $f \triangle g$ is not peripherally continuous at $x$ from the left and by Theorem $4, f \notin \mathcal{M}_{\triangle}\left(\mathrm{b} \mathcal{C}_{\text {ap }}\right)$.

Similarly we proceed if $C$ is not non-degenerate at $x$ from the right. 
(iii) $\Rightarrow$ (i). Let $g \in \mathcal{C}_{\text {ap }}$. Fix an $x \in \mathbb{R}$ and an $\varepsilon>0$. Let $C$ be as above and $D=g^{-1}([g(x)-\varepsilon, g(x)+\varepsilon])$. Since $C$ is non-degenerate at $x$ from the left, we can find a sequence $a_{n} \nearrow x$ such that

$$
\lim _{n \rightarrow \infty} \frac{\left|\left[a_{n}, x\right] \cap C\right|}{x-a_{n}}=2 c>0 .
$$

Let $\eta \in(0, \varepsilon)$ be such that

$$
\frac{\left|\left[a_{n}, x\right] \cap C\right|}{x-a_{n}}>c \quad \text { if } a_{n}>x-\eta, n \in \mathbb{N},
$$

and

$$
\frac{|[t, x] \cap D|}{x-t}>1-c \quad \text { if } t \in(x-\eta, x) .
$$

Then $\left|\left[a_{n}, x\right) \cap C \cap D\right|>0$ for each $n \in \mathbb{N}$ with $a_{n}>x-\eta$. Hence $f \triangle g$ is peripherally continuous at $x$ from the left.

Similarly we can prove that $f \triangle g$ is peripherally continuous at $x$ from the right. This implies that the graph of $f \triangle g$ is connected, so $f \in \mathcal{M}_{\triangle}\left(\mathcal{C}_{\mathrm{ap}}\right)$.

Now we turn to the family $\mathcal{D}$.

Proposition 7. Let $f \in \mathcal{B}_{1}$. Then the following conditions are equivalent:

(i) $f \in \mathcal{M}_{\triangle}(\mathcal{D})$,

(ii) $f \in \mathcal{M}_{\triangle}\left(\mathrm{b} \mathcal{D} \mathcal{C}_{0}\right)$,

(iii) for each $x \in \mathbb{R}$ and each $\varepsilon>0$ the complement of $f^{-1}([f(x)-\varepsilon$, $f(x)+\varepsilon])$ is bilaterally porous at $x$.

Proof. The implication (i) $\Rightarrow$ (ii) is obvious.

(ii) $\Rightarrow$ (iii). Suppose that for some $x \in \mathbb{R}$ and some $\varepsilon \in(0,1), \mathbb{R} \backslash C$ is not porous at $x$ from the left, where $C=f^{-1}([f(x)-\varepsilon, f(x)+\varepsilon])$. Then there exists a sequence $x_{n} \nearrow x$ such that $x_{n} \notin C$ for each $n \in \mathbb{N}$ and

$$
\lim _{n \rightarrow \infty} \frac{x_{n+1}-x_{n}}{x-x_{n}}=0 .
$$

For each $n \in \mathbb{N}$ set $A_{n}=\left[x_{n}, x_{n+1}\right] \backslash C$. We consider two cases:

- Assume that int $A_{n} \neq \emptyset$ for each $n \in \mathbb{N}$. Let $y_{n} \in\left(a_{n}, b_{n}\right) \subset \operatorname{int} A_{n}$. For each $n \in \mathbb{N}$ put $J_{n}=\left[y_{n}, y_{n+1}\right]$, define a continuous function $g_{n}$ by

$$
g_{n}(t)= \begin{cases}0 & \text { if } t \leq y_{n} \text { or } t \geq y_{n+1}, \\ 1 & \text { if } t \in\left[b_{n}, a_{n+1}\right], \\ \text { linear } & \text { in }\left[y_{n}, b_{n}\right] \text { and }\left[a_{n+1}, y_{n+1}\right],\end{cases}
$$

and set $r_{n}=\int_{J_{n}} g_{n}$. Choose a sequence $\left(t_{n}\right)$ according to Lemma 5 . Define

$$
g(t)= \begin{cases}0 & \text { if } t \leq a_{1} \text { or } t \geq x, \\ t_{n} \varepsilon g_{n}(t) & \text { if } t \in J_{n}, n \in \mathbb{N} .\end{cases}
$$


Then clearly $g$ is bounded and continuous except possibly at $x$, and it is continuous at $x$ from the right. Moreover, for each $t \in J_{n}$,

$$
\begin{aligned}
\frac{1}{x-t}\left|\int_{t}^{x} g\right| & \leq \frac{1}{x-t}\left(\left|\sum_{k=n+1}^{\infty} \int_{J_{k}} g\right|+\int_{J_{n}}|g|\right) \\
& \leq \frac{3 \varepsilon \sup \left\{\left|J_{k}\right|: k \geq n\right\}}{x-t} \\
& \leq 3 \varepsilon \sup \left\{\frac{x_{k+2}-x_{k}}{x-x_{n+2}}: k \geq n\right\} \\
& \leq 3 \varepsilon \sup \left\{\frac{x_{k+2}-x_{k}}{x-x_{k}} \cdot\left(1-\frac{x_{k+2}-x_{k}}{x-x_{k}}\right)^{-1}: k \geq n\right\} .
\end{aligned}
$$

So by (1), $g$ is a derivative, whence $g \in \mathrm{b} \mathcal{D} \mathcal{C}_{0}$. But for each $t \in\left[a_{1}, x\right)$ either $t \notin \bigcup_{n=1}^{\infty}\left(a_{n}, b_{n}\right)$, so that $|g(t)|=|g(t)-g(x)|=\varepsilon$, or $t \in \bigcup_{n=1}^{\infty}\left(a_{n}, b_{n}\right)$, in which case $t \notin C$ and $|f(t)-f(x)|>\varepsilon$. It follows that $f \triangle g$ is not peripherally continuous at $x$ from the left and $f \notin \mathcal{M}_{\triangle}\left(\mathrm{bDC} \mathcal{C}_{0}\right)$.

- Assume that $\operatorname{int} A_{n}=\emptyset$ for some $n \in \mathbb{N}$. Then $C$ is residual in $\left[x_{n}, x_{n+1}\right]$. Define $\varepsilon^{\prime}=\left(\left|f\left(x_{n}\right)-f(x)\right|-\varepsilon\right) / 2$ and $C^{\prime}=f^{-1}\left(\left[f\left(x_{n}\right)-\varepsilon^{\prime}\right.\right.$, $\left.\left.f\left(x_{n}\right)+\varepsilon^{\prime}\right]\right)$. Then $C^{\prime}$ is a $G_{\delta}$ set and $C^{\prime} \cap C=\emptyset$, so $C^{\prime}$ is nowhere dense in $\left[x_{n}, x_{n+1}\right]$. Hence the complement of $C^{\prime}$ is not porous at $x_{n}$ from the right and for every interval $J, \operatorname{int}\left(J \backslash C^{\prime}\right) \neq \emptyset$. Now proceed as in the previous case, using $x_{n}$ instead of $x$ and $\varepsilon^{\prime}$ instead of $\varepsilon$.

Similarly we proceed if $\mathbb{R} \backslash C$ is not porous at $x$ from the right.

(iii) $\Rightarrow$ (i). Let $g \in \mathcal{D}$ and let $G$ be its primitive. Fix an $x \in \mathbb{R}$ and an $\varepsilon>0$. Define $C$ as above. Since $\mathbb{R} \backslash C$ is porous at $x$ from the left, we can find a sequence $a_{n} \nearrow x$ such that $\left[a_{2 n-1}, a_{2 n}\right] \subset C$ and

$$
\lim _{n \rightarrow \infty} \frac{a_{2 n}-a_{2 n-1}}{x-a_{2 n-1}}=2 c>0 .
$$

Let $\eta \in(0, \varepsilon)$ be such that for each $t \in(x-\eta, x)$,

$$
\frac{a_{2 n}-a_{2 n-1}}{x-a_{2 n-1}}>c \quad \text { if } a_{2 n-1}>x-\eta, n \in \mathbb{N},
$$

and

$$
\left|\frac{G(t)-G(x)}{t-x}-g(x)\right| \leq \frac{c \varepsilon}{2} \quad \text { if } t \in(x-\eta, x) .
$$

Then for each $n \in \mathbb{N}$ with $a_{2 n-1}>x-\eta$, we get

$$
\left|\frac{G\left(a_{2 n}\right)-G\left(a_{2 n-1}\right)}{a_{2 n}-a_{2 n-1}}-g(x)\right| \leq \frac{2 x-a_{2 n}-a_{2 n-1}}{a_{2 n}-a_{2 n-1}} \cdot \frac{c \varepsilon}{2}<\varepsilon .
$$

Hence there exists a $t \in\left[a_{2 n-1}, a_{2 n}\right]$ such that $|g(t)-g(x)| \leq \varepsilon$. But since $\left[a_{2 n-1}, a_{2 n}\right] \subset C$, also $|f(t)-f(x)| \leq \varepsilon$. It follows that $f \triangle g$ is peripherally continuous at $x$ from the left. 
Similarly we can prove that $f \triangle g$ is peripherally continuous at $x$ from the right. This implies that the graph of $f \triangle g$ is connected, so $f \in \mathcal{M}_{\triangle}(\mathcal{D})$.

Remark. By Proposition 7 , we get $\mathcal{M}_{\triangle}\left(\mathcal{D} \mathcal{C}_{\text {a.e. }}\right)=\mathcal{M}_{\triangle}(\mathcal{D})$. It turns out that the analogous result does not hold for the family $\mathcal{C}_{\text {ap }}$.

Proposition 8. Let $f \in \mathcal{B}_{1}$. Then the following conditions are equivalent:

(i) $f \in \mathcal{M}_{\triangle}\left(\mathcal{C}_{\text {ap }} \mathcal{C}_{\text {a.e. }}\right)$,

(ii) $f \in \mathcal{M}_{\triangle}\left(\mathrm{b} \mathcal{C}_{\text {ap }} \mathcal{C}_{0}\right)$,

(iii) for each $x \in \mathbb{R}$ and each $\varepsilon>0$ the closure of $f^{-1}([f(x)-\varepsilon, f(x)+\varepsilon])$ is bilaterally non-degenerate at $x$.

Proof. The implication (i) $\Rightarrow$ (ii) is obvious.

(ii) $\Rightarrow$ (iii). Suppose that for some $x \in \mathbb{R}$ and some $\varepsilon>0, \operatorname{cl} C$ is not non-degenerate at $x$ from the left, where $C=f^{-1}([f(x)-\varepsilon, f(x)+\varepsilon])$. For each $n \in \mathbb{N}$ set $x_{n}=x-1 / n, I_{n}=\left[x_{n}, x_{n+1}\right]$ and $A_{n}=\operatorname{int} I_{n} \backslash \operatorname{cl} C$, find a closed set $B_{n} \subset A_{n}$ such that $\left|A_{n} \backslash B_{n}\right| \leq\left|A_{n}\right| / n$, and find a continuous function $g_{n}$ such that $0 \leq g_{n} \leq 1$ on $\mathbb{R}, g_{n}=1$ off $A_{n}$ and $g_{n}=0$ on $B_{n}$. Define

$$
g(t)= \begin{cases}0 & \text { if } t \leq x_{1} \text { or } t \geq x \\ \varepsilon g_{n}(t) & \text { if } t \in I_{n}, n \in \mathbb{N} .\end{cases}
$$

Then clearly $g \in \mathrm{bC}_{0}$. Repeating the argument of Proposition 6 one can see that $g \in \mathcal{C}_{\text {ap }}$ and $f \triangle g$ is not peripherally continuous at $x$ from the left. Hence $f \notin \mathcal{M}_{\triangle}\left(\mathrm{bC}_{\text {ap }} \mathcal{C}_{\text {a.e. }}\right)$.

Similarly we proceed if $\operatorname{cl} C$ is not non-degenerate at $x$ from the right.

(iii) $\Rightarrow\left(\right.$ i). Let $g \in \mathcal{C}_{\text {ap }} \mathcal{C}_{\text {a.e. }}$. Fix an $x \in \mathbb{R}$ and an $\varepsilon>0$. Let $C$ be as above and $D=g^{-1}((g(x)-\varepsilon, g(x)+\varepsilon))$. Since cl $C$ is bilaterally non-degenerate at $x$, we can find a sequence $a_{n} \nearrow x$ such that

$$
\lim _{n \rightarrow \infty} \frac{\left|\left[a_{n}, x\right] \cap \operatorname{cl} C\right|}{x-a_{n}}=2 c>0 .
$$

Let $\eta \in(0, \varepsilon)$ be such that

$$
\frac{\left|\left[a_{n}, x\right] \cap \operatorname{cl} C\right|}{x-a_{n}}>c \quad \text { if } a_{n}>x-\eta, n \in \mathbb{N},
$$

and

$$
\frac{|[t, x] \cap D|}{x-t}>1-c \quad \text { if } t \in(x-\eta, x) .
$$

Then for each $n \in \mathbb{N}$ with $a_{n}>x-\eta$, we get

$$
\left|\left(a_{n}, x\right) \cap \operatorname{cl} C \cap D\right|=\left|\left(a_{n}, x\right) \cap \operatorname{cl} C \cap \operatorname{int} D\right|>0 .
$$

(We used the fact that $D$ is a.e.-open.) Hence $\left(a_{n}, x\right) \cap C \cap D \neq \emptyset$, so $f \triangle g$ is peripherally continuous at $x$ from the left. 
Similarly we prove that $f \triangle g$ is peripherally continuous at $x$ from the right. This implies that the graph of $f \triangle g$ is connected and that $f \in$ $\mathcal{M}_{\triangle}\left(\mathcal{C}_{\text {ap }} \mathcal{C}_{\text {a.e. }}\right)$.

Proposition 9. $\mathcal{M}_{\triangle}\left(\mathcal{C}_{\text {ap }} \mathcal{C}_{\text {a.e. }}\right) \backslash \mathcal{M}_{\triangle}\left(\mathcal{C}_{\text {ap }}\right) \neq \emptyset$.

Proof. For each $n \in \mathbb{N}$ set $a_{n}=1$ and find a nowhere dense perfect set $F_{n}$ such that inf $F_{n}=a_{n+1}, \sup F_{n}=a_{n}$ and $\left|F_{n}\right|=(1-1 / n)\left(a_{n}-a_{n+1}\right)$. Observe that

$$
0 \in d \text {-int }\left((-\infty, 0] \cup \bigcup_{n=1}^{\infty} F_{n}\right) .
$$

Fix an $n \in \mathbb{N}$. Let $\left\{E_{n, k}: k \in \mathbb{N}\right\}$ be the family of all components of $\left(a_{n+1}, a_{n}\right) \backslash F_{n}$. For all $k \in \mathbb{N}$ if $E_{n, k}=\left(b_{n, k}-c_{n, k}, b_{n, k}+c_{n, k}\right)$, then let $f_{n, k}$ be a continuous function such that $f_{n, k}\left(b_{n, k}\right)=1$ and $f_{n, k}(x)=0$ if $\left|x-b_{n, k}\right| \geq \max \left\{c_{n, k}, c_{n, k}^{2}\right\}$. Define

$$
f(x)= \begin{cases}f_{n, k}(x) & \text { if } x \in E_{n, k}, n, k \in \mathbb{N}, \\ 1 & \text { if } x \leq 0, \\ 0 & \text { otherwise. }\end{cases}
$$

Then evidently $f$ is approximately continuous on $\mathbb{R} \backslash\{0\}$ and it is continuous at 0 from the left, so at these points condition (iii) of Proposition 8 is satisfied. Fix an $\varepsilon \in(0,1)$ and set $C=f^{-1}([1-\varepsilon, 1+\varepsilon])$. Then $C \cap \bigcup_{n=1}^{\infty} F_{n}=$ $\emptyset$ and since $C \cap E_{n, k} \neq \emptyset$ for all $n, k \in \mathbb{N}$, we have $\operatorname{cl} C \supset \bigcup_{n=1}^{\infty} F_{n}$. Hence by (2), $f$ does not satisfy condition (iii) of Proposition 6 and it does satisfy condition (iii) of Proposition 8, which completes the proof.

Remark. It is easy to see that the function constructed in the proof of Proposition 9 is discontinuous on a set of positive measure. This leads to conjecturing that for functions from $\mathcal{C}_{\text {a.e. }}$ the proposition analogous to Proposition 7 holds. This is indeed true.

Proposition 10. $\mathcal{M}_{\triangle}\left(\mathcal{C}_{\text {ap }} \mathcal{C}_{\text {a.e. }}\right) \cap \mathcal{C}_{\text {a.e. }}=\mathcal{M}_{\triangle}\left(\mathcal{C}_{\text {ap }}\right) \cap \mathcal{C}_{\text {a.e. }}$.

Proof. The inclusion " $\supset$ " is obviously satisfied.

Let $f \in \mathcal{M}_{\triangle}\left(\mathcal{C}_{\text {ap }} \mathcal{C}_{\text {a.e. }}\right) \cap \mathcal{C}_{\text {a.e. }}, x \in \mathbb{R}$ and $\varepsilon>0$. Set $C=f^{-1}([f(x)-\varepsilon$, $f(x)+\varepsilon]$ ). By Proposition $8, \operatorname{cl} C$ is bilaterally non-degenerate at $x$. Since $t \in C$ implies $|f(t)-f(x)| \leq \varepsilon$, it follows that for every $t \in \operatorname{cl} C$, if $t$ is a point of continuity of $f$, then $|f(t)-f(x)| \leq \varepsilon$. Hence $|\operatorname{cl} C \backslash C|=0$ and $C$ is bilaterally non-degenerate at $x$, i.e., condition (iii) of Proposition 6 is satisfied.

\section{References}

[1] A. M. Bruckner, Differentiation of Real Functions, Lecture Notes in Math. 659, Springer, Berlin, 1978. 
[2] A. M. Bruckner, J. Mařík and C. E. Weil, Baire one, null functions, in: Contemp. Math. 42, Amer. Math. Soc., 1985, 29-41.

[3] C. Goffman, C. J. Neugebauer and T. Nishiura, Density topology and approximate continuity, Duke Math. J. 28 (1961), 497-506.

[4] K. Kuratowski, Topology, Vol. I, Academic Press, New York, 1966.

[5] J. Lukeš, J. Malý and L. Zajíček, Fine Topology Methods in Real Analysis and Potential Theory, Lecture Notes in Math. 1189, Springer, Berlin, 1986.

[6] A. Maliszewski, Characteristic functions and products of bounded derivatives, Proc. Amer. Math. Soc., to appear.

[7] C. J. Neugebauer, On a paper by M. Iosifescu and S. Marcus, Canad. Math. Bull. 6 (1963), 367-371.

[8] R. J. O'Malley, Approximately continuous functions which are continuous almost everywhere, Acta Math. Acad. Sci. Hungar. 33 (1979), 395-402.

[9] G. Petruska and M. Laczkovich, Baire 1 functions, approximately continuous functions and derivatives, ibid. 25 (1974), 189-212.

[10] Z. Zahorski, Sur la première dérivée, Trans. Amer. Math. Soc. 69 (1950), 1-54.

\author{
DEPARTMENT OF MATHEMATICS \\ PEDAGOGICAL UNIVERSITY \\ CHODKIEWICZA 30 \\ 85-064 BYDGOSZCZ, POLAND \\ E-mail: WSPB05@PLTUMK11.BITNET
}

Received 31 March 1993;

in revised form 28 March 1994 